The Journal of

Mathematics and Computer Science

Available online at

\title{
http://www.TJMCS.com
}

The Journal of Mathematics and Computer Science Vol .2 No.2 (2011) 284-294

\section{Surveying Robot Routing Algorithms with Data Mining Approach}

\author{
Rouhollah Maghsoudi 1, ${ }^{*}$, Somayye Hoseini' ${ }^{2}$, Yaghub Heidari ${ }^{3}$ \\ Department of Computer, Nour Branch, Islamic Azad University, Nour, Iran \\ Payame Noor University of Shahrerey,Tehran, Iran, sce.hoseyny@gmail.com \\ Department of Electrical, Nour Branch, Islamic Azad University, Nour, Iran
}

Received: July 2010, Revised: October 2010

Online Publication: January 2011

\begin{abstract}
Data mining knowledge in response to technological advances in various Rmynh, foot arena is built there. Data Mining face a different situation that the data size is large and we want to build a small model and not too complicated and yet the data as well as describe. Necessity to use data analysis to reduce the amount and the huge volume of information. One important and practical issues in the world of machine intelligence and is robotics robots routing. Robot router has obstacle detection and how to deal with the decision with obstacle. For routing, algorithms including probabilistic methods (filtering particulate), evolutionary algorithms such as genetic, ants social and optimization particle mass, neural methods - Fuzzy, inequality of matrix method based on gradient methods combined sensor information, etc. There are data mining methods in the years 2010-2008 as a technique for routing and a complete robot has been used and still is in progress. Overview of the methods in the paper mentioned in various articles since 2000 has so far. Although many data mining methods include, but mentioned in this article with specific literature data mining will deal with the routing problem.
\end{abstract}

\footnotetext{
$1,{ }^{*}$ Corresponding Author.

E-mail address: rcemaghsoudy@yahoo.com (Phone: +98 122625 4590)

${ }^{2}$ Computer Department of Shahrerey Payame Noor

${ }^{3}$ Electrical Department of Islamic Azad university of Nour (Phone: +98 1226254590 )
} 
Keywords: data mining, robot routing.

\section{Introduction}

Routing for a mobile robot, the robot path by passing it through without collision with obstacles in the environment to reach their destination of origin. Movable robot for tasks such as locate the robot, routing and no need to deal with obstacles accurate model with a degree of confidence from its surrounding. In methods and algorithms presented so far most of the data used for the robot and the robot were known among the data available, the best option would decide to adopt.

With significant increase in volume of data stored on the need for better methods, faster and cheaper to analyze them was felt. And if this purpose can't be efficient and effective mechanism to extract knowledge from huge volumes of data design will, then all the data available in the world will be worthless. A number of scientists discovered the necessity of such a need and thus was born the science data mining. That some of these leading researchers are: h.Mannila, p.Smith, G. Djoryorski.

On the other hand the hypothesis that users usually raised and then count based on reports prove or reject the hypothesis data. While today is the need for ways to pay the so-called knowledge discovery with minimal user intervention and automatically logical patterns and relationships to express.

Data mining is one of the most important methods by which useful patterns in data with minimal user intervention are known and available information of users and analysts to make decisions based on their vital organizations to adopt.

Half-term data mining process automatically analyze large databases to find useful patterns can be applied. Data mining is the process involves three steps are:

1. Initial excavation

2. Construction model or gain credit with the help of pattern recognition / approval,

3. Operation

Step 1: search. Usually this order with data preparation will be done which may include data cleansing, data conversion and election the sub-set of fields is with massive volume of variable (fields). Then according to nature analytical predictions, this order to precaution model simple or comments to identify variables and determine the complexity of models for use in the next step requires.

Step 2: Construction and verification of model validity. This stage to review different models and selecting the best model predicting the efficiency of the deals. Several techniques were developed to achieve this goal. And "competitive evaluation of models" were named. For this purpose different models used identical data collection until be compared their efficiency, then the model that have the best performance, is chosen. This technique include: Bagging, Boosting, Stacking and Meta-learning.

Step 3: exploit. Last step before a model that has been selected, the work in new data until expectant`s precaution outs.

About Good benefits of data mining process quick lead against the received data and is not guided. Data Mining in Business, the largest group to use the techniques and concepts of data mining form, participation is. And its application to companies telecom AT \& $\mathrm{T}$ and oil companies like MOBIL OIL and insurance (analysis of claims and predict the amount of insurance purchase by new customers) and medical and banking and so is the volume of millions of data takes and processes[1,6].

This paper reviews the algorithm and data mining models that are used to pay a robot routing.

\section{Evolutionary algorithms and their application in routing}


In general, selection and design optimization problems in many scientific and technical make the best possible answer in a product or special circumstances are. For example, suitable products in the areas of technical and engineering related to design accurate and optimum`s shape, size and parts product it is. In the area of Mechatronics (Robotics) optimum 's path can move a robot arm pointed. Generally in all matters looking for the best possible answer we get back, but among all the solutions and answers which is optimal? Therefore, the importance of optimal selection and optimization in all the issues we realized: our goal is that space may be looking for answers Look for the best answer.

Evolutionary algorithm technique to implement mechanisms such as reproduction, mutation, this combination (merger), natural selection (a process in which individuals with desirable characteristics more likely to produce the next people are used to. The desirable features in general are becoming the next generation.), and survival is most qualified. Evolutionary computation often includes algorithms optimization past discovery such:

1 - Evolutionary algorithms which includes (genetic algorithms, evolutionary programming, Genetic Programming)

2- Intelligence, a group that (optimization particle group, and ant bee)

2-1 genetic algorithms: the idea as one of the methods of random optimization by john Holland was invented in 1967, and then this method attempts Goldberg in 1989 and found his places today through the capabilities of their place among other methods are. Specific type of genetic algorithms evolutionary algorithms that learn biology techniques such as inheritance and mutation patrol uses. Genetic Algorithm with all optimization methods are different. Genetic algorithms search technique based on computer algorithms and optimization based on the structure of genes and chromosomes are. Robot to imitate the behavior of creatures and GA to mimic biological motion. The aim of this investigation with GA algorithms move the robot arm to be optimized so that the barriers and stop modes impossible not to encounter. In cases where the answers too complicated or inactivity or if the answers together and available with or if you require a means of exploration to find new ways we can use genetic algorithms. The most important GA applications in science: machine learning, robotics, management planning, etc...

\section{Routing with genetic algorithm}

As you know, the robot can imitate the behavior of creatures and GA can mimic biological life. Since much research in the field of Robot trajectory using GA has been such that it can be to Davidor in 1991 and in 1996 Pack noted.

The aim of this research, for example, moves a robot arm is optimized so that the obstacles facing mode are not impossible. So far, very complex methods using parallel GA algorithm in 1995 by Chambers in this regard has been presented. As another example, in the race war robot for the first time (Yao 1995) of GA was used. Basically, the path moves a robot-related position, direction; velocity and acceleration components are vast. In this example, our robot with Link and 2 degrees of freedom is considered. Obstacle to a curve that has become an oval-shaped area surrounded by and The Oval where the robot arm can not pass it. For example, assume four obstacle space desired that there be only three or four pieces of the middle point of the line are allowed to be the shortest path from the beginning to the end of the path are bring. in solving this problem from binary GA was used. Robot arm to move through barriers in the workspace is shown in shape 1. 


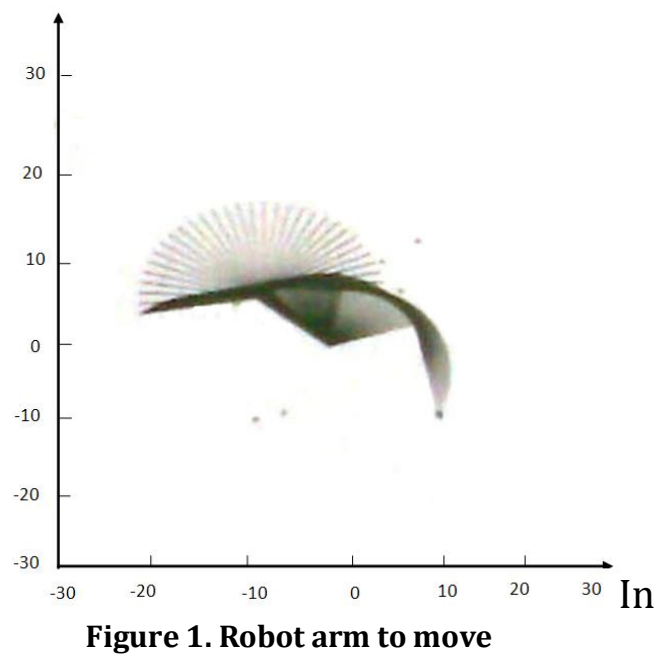

2-1-2 Ant algorithm (Aco): the first time by Dorigo, Maniezzo and Colorni as innovative methods for solving hard optimization problems was introduced compounds. An optimization algorithm that can learn ultra discovery approximate solutions for optimization problems to find a combination problem. Aco ant in artificial moving on the problem graph solutions make and imitate real ants on a graph, instead of putting artificial wheel so that the artificial ants coming to find better solutions $[2,5]$.

\section{Routing with Ant Algorithm}

Suppose, a picture of where the robot has been given to the robot and the robot has the job after the image processing and recognition of non-crossing which part is the intelligent diagnosis which give direction to achieve target is shorter. Under such circumstances, the first image is divided into squares, and what we need more precision the number of squares can be larger. After image processing is unknown due to obstacles the robot on which a network of homes can not go on what can go home. For example, shape 2 , which was taken from the surface consider. This image first network $10 * 10$ is divided and the process has been known for the robot on which a house can not go (figure 3). Now the image processed robot should go, where is (R) of the shortest path to Target (T) and go for a smart way to choose the best algorithm ant uses.

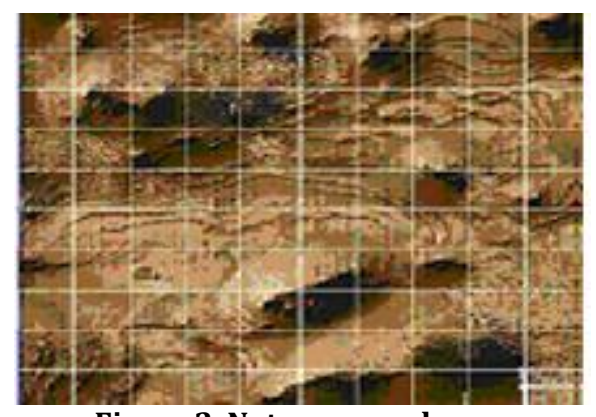

Figure 2. Not processed 


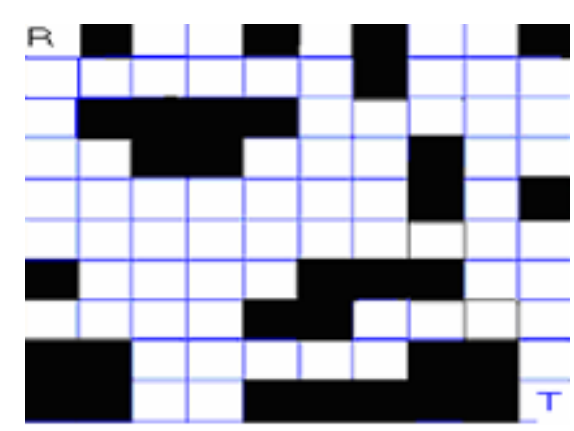

Figure 3. Processed

The algorithm is as follows:

1 - set initial wheel on each home.

2 - Placed on the origin of ants

3 - Create a response for each ant

4 - wheel when the best response to

choose the best answer

5 - Czech bet to end

$\mathrm{N}$ ants do this put on the origin of the ants, one after another began to move and while it brings the answer, the amount of pheromone that the ants themselves rather than pass under a formula is calculated. We know that an ant at each step, more likely to choose a path that has been shed on pheromone is greater. Now suppose n`s ant,i`s house is located and wants to choose next to the house. In this regard, we know that ants homes that already have or that they stand in is not selected. International House of $\mathrm{k}$ let them have chosen, the probability that ants have to choose the house $\mathrm{j}$ is calculated according to formula. After n`s ant arrived at its destination, turn to check if the algorithm reaches the end

2-1-3 optimize particle group (Pso): The idea for the first time by Kenedy \& oberhart was introduced in 1995, an evolutionary computing algorithm inspired by nature and is based on repetition. This algorithm inspired social behavior of animals, mass movement of birds is. Hence that, Pso with a random initial population matrix begins like many other evolutionary algorithms like genetic algorithms and the algorithm competitive colonization are continuous. Pso algorithm of a certain number of particles is formed by random initial value and is two values for each particle position and velocity are defined. These particles form repeated in $n$ dimensional space, the problem moves with the calculated value as an optimality criterion to measure the new options may be to seek it.

Advantages of this method are that the implementation of this algorithm is simple and requires little set parameters and to optimize complex functions with a large number of minimum costs is local [8].

\section{PSO Algorithm}

Subjects discussed in this section Rummy can be summarized in the following algorithm:

1. Initialize

2. For each sample in the current population, competency level of each sample using the formula is calculated and best value is stored as best.

3. The best answer is clear the current population

4. For each sample population and repeat the following steps:

A) The sample rate is set.

B) The location of each sample is updated

Go to Step 2 and we repeat... 
Each time step, the evolutionary algorithm several times (generation) is repeated. In site selection across the position of the target search space is not available and we can only place to find the robot sensors (target) can be used

2-1-4 bee algorithm (BA): BA first by PHAM and colleagues was presented. Relatively new branch of nature-inspired collective intelligence that is called the behavior of some insects to develop effective discussions are focused on exploratory and can problem solve imitate insects moving. Bee algorithm a new topic you are exploratory discussions. BA (bee algorithm) tries to bee behavior in modeling to find food. Several mechanisms such as bee waggle dance (How to move the bees to move in different directions) to achieve the desired food to research. This them a good candidate for solving optimization problems has. Bee social behavior can be as dynamic systems gather information from the environment and adjust their behavior accordingly be considered. BA applications in solving complex traffic and transportation engineering problems, allocation of resources to solve problems, Dynamic allocation of Internet services and for routing in telecommunication networks, etc

\section{Routing with Bees Algorithm}

Algorithm bee anywhere in the space parameter (consists of responses may be ) as a source of food (goal) under review offers. "Bee of Watch" ( brokers simulated ) (robot which environments are) a (Random ) space, the answers are simple and by the merit function has had quality opportunities to the report (which are what the situation, there are close to the goal or not?) simplified answers are ranked, and other bees (robots) that forces a new space in response to their surroundings to find out the top places to search (the garden (environment) is called) as a selective algorithm to find another garden to point maximize the merit function will search. (Environment which goal is more suitable)[3].

\section{Study of neural network algorithms and their application in routing}

Neural Networks: In recent years witnessed continuous motion, from purely theoretical research to applied research, especially in the field of information processing for issues for which a solution is not available or are not easily have been resolved. Considering the growing interest in this development Theodoric intelligent dynamic systems, which are based on experimental data, has been created. Neural networks in these systems are dynamic, with the experimental data processing or knowledge lies beyond the data transferred to the network structure. That's why these systems intelligent to say, why, based on Numerical calculations on data or examples to learn the general rules. Model-based neural network after training based on available data, the ability and predictability can find and create a black box models, mapping between input and output data through patterns recorded by during their practical training. A neural network-based information processing techniques methods such as biological nervous systems and brains processed information. Fundamental concept of neural network structure, information processing system that a large number of processing units, neurons) associated with the networks (formed. Biological neuron or neurons, the nervous system manufacturer unit in humans. Neural networks used particular why are they an effective tool for modeling large and complex issues that they may be hundreds of variables for predicting the interactions are very present. (Biological neural networks automatically incomparable are more complicated.) Neural networks problems can be classified or surmises return (in which the output variable is continuous) can be used. a neural network with an inner layer begins in which each node is assigned a predictive variable. The nodes into a number of nodes in the hidden layer are connected. Nodes in the hidden layer nodes can someone or other hidden layer output can be connected to a layer. Output layer includes one or more variables is the answer

Field neural network applications in the following topics:

- Unknown correlation between features and value of desirable variables to decide issues where solutions to problems is unknown. 
- Issues that do not have the solution algorithm.

- where data is incomplete.

The main advantage of neural networks, their extraordinary ability to learn and also their stability against small disturbances is input. As a result, our focus is the fact that the calculations correctly selected the right network, the main factor in ensuring successful performance [4].

\section{Routing with Neural Networks}

For example, we plan to move the surface smooth for a robot with two degrees of freedom through some barriers polygon motionless with network neural Hopfield review to. The robot can only deform to find rather than during or turn on a flat surface be. Motion planning problem can be completed by the display (Display a full calculate)in the free configuration space efficiently resolved. This approach, with configuration space analyze cells trapezoidal network and build road map may be released from cells is. Classical path planning methods, including the road map, and analyze cells using global potential field to search for possible paths in the workspace may have. These model only static environments that are complex to handle.

Hopfield network concept in problem traveling salesman Badger first proposed by Hopfield and tank in 1985 and then by routing problem with this approach was followed. Hopfield network and its capability of providing intrinsic mass calculations required to search for solutions to optimization problems are large (2008-1993).

First use of network Hopfield, making road map is a graph. And using such analysis techniques and probabilistic methods and so we can position the obstacles in the map to show. The road map analysis, as a collection of discrete cells and planned to do between making connection graph using the adjacency relations between cells, is presented. Those adjacent cells can be directly below the form to move between them (according figure4).

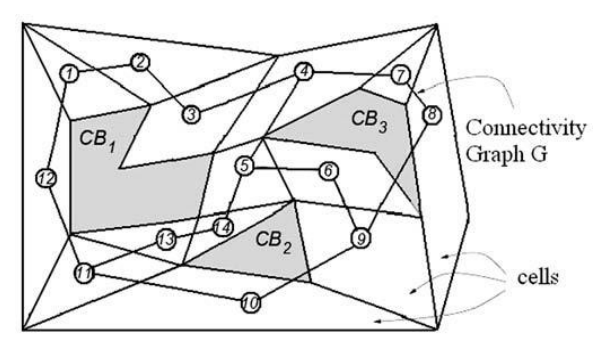

Figure 4.network Hopfield

\section{Hopfield architecture for optimization}

Figure5 show a following a complete system of $\mathrm{n}$ continuous nerve shows. 


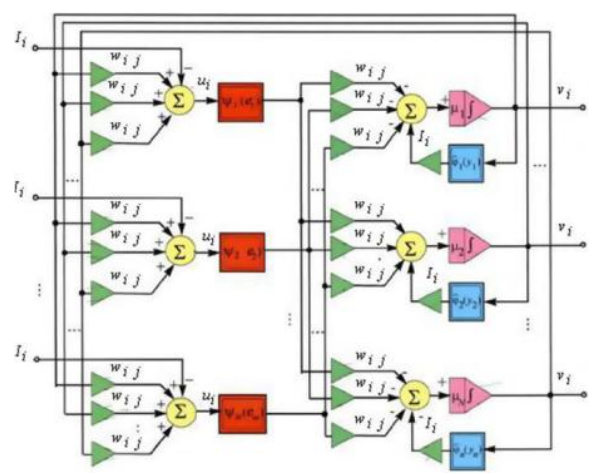

Figure 5. Hopfield architecture

Nerve $i, u_{i}$ is an internal situation, and the outer surface is $v_{i}$. Is a range between zero and one.. $u_{i}$ internal status of a current-voltage (or neutralization threshold) specified weight set with $l_{i}$ and production of nerve other weight is associated with nerve connections to the power of $\mathrm{i}$ to $\mathrm{j}$ to specify the device to $w_{i j}$. The relationship between the internal situation of a nerve and its external surface with an activation function $\left(u_{i}\right)$ is determined that the range is below zero and above one. And function as the activation function is defined. That continuously increasing and is diagnosed.

\section{Run Hopfield}

A Hopfield network can find local optimal solutions of energy function to use, which may to a local minimum solution of the optimization problem becomes.

$G=(V, E)$ a directed graph in which, respectively, $V=(1 \ldots n)$ and $E$ a set of nodes and edges are. Value

Assignment, $\mathrm{P}=1$..., $\mathrm{P}$, cost vector and $\mathrm{E}=(\mathrm{i}, \mathrm{j}) . \mathrm{S}, \mathrm{d} \in \mathrm{N}$ are source and destination nodes.

Minimize $z^{p}=\sum_{(i, j) \in A} c_{i, j}^{p} x_{i, j} \quad p=1, \ldots \ldots . p$

$x_{i, j} \in\{0,1\}$

Using formulas Hopfield is used:

Since the computer simulations, working on a matrix with smaller scale compared to larger scale, are easier. We have a new variable called instead for $\mathrm{i}, \mathrm{j}=1 \ldots \mathrm{N}$ for membership in this index are defined Let. And we use the following function:

$$
\begin{aligned}
& f:\{1, \ldots \ldots, n\} \times\{1, \ldots \ldots . n\} \rightarrow\left\{1, \ldots \ldots, n^{2}\right\} \\
& f(i, j)=n(i-1)+j .
\end{aligned}
$$

Can be shown that a function $\mathrm{f}$ is one to one. So our goals for this function can be used. sets that we or equivalently in the formula instead of the multi-objective shortest path problem put. And is as follows:

$$
\begin{aligned}
& E(v)=\sum_{p=1}^{p} \theta_{p}\left(\sum_{l=1}^{n^{2}} c_{l}^{p} \cdot v_{l}-z_{*}^{p}\right)^{2}+\alpha_{1} \sum_{l=1}^{n^{2}} \gamma_{l} \cdot v_{l}+\alpha_{2} \times \sum_{i \neq s, d}\left(\sum_{l=n(i-1)+1}^{n i} v_{l}-\sum_{l=i}^{n(n-1)+i} v_{l}\right)^{2} \\
& +\alpha_{3}\left(\sum_{l=n(s-1)+1}^{n s} v_{l}-1\right)^{2}+\alpha_{4}\left(\sum_{l=d}^{n(n-1)+d} v_{l}-1\right)^{2}+\alpha_{5}\left(1-v_{n(d-1)+s}\right)+\alpha_{6} \sum_{l=1}^{n^{2}} v_{l}\left(1-v_{l}\right)
\end{aligned}
$$

Using this formula comes to the network using the shortest path algorithm can Dayjestra using to bring costs obtained (according figure 6) [7, 9]. 


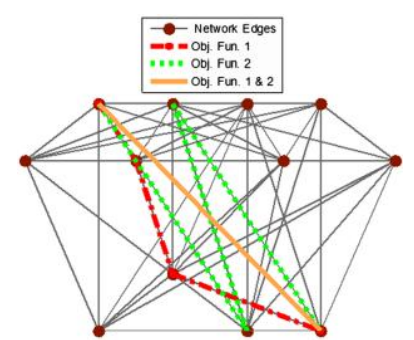

Figure 6. Network formed using the relation 3

\section{Neural Fuzzy Networks}

Simple definition of neural networks - those fuzzy systems are a combination of techniques from neural networks and fuzzy logic as a complementary use. Preferred method and logic combination of the two mentioned together for their benefits and flaws of each method are elimination. Using a mathematical model in complex systems, especially systems with nonlinear behavior or systems that communicate enough information about their input and output is not very difficult. For this reason, many scholars regard to the use of networks and fuzzy logic Haysby have focused on. Neural networks for information systems or a system Thtkntrl must be learned are extracted. While fuzzy logic often their information from experts to get. Ideally these two sources should be combined with each other, for example, rules can be one of these two methods learned and can be set by other methods. Such a system capable of matching the one hand the ability to learn and how reasoning, inference and decision making, and its capacity for another correction, organize, develop, and decision making stems from their flexibility. Such a system features in solving various problems it is very excellent. Thought that the neural fuzzy systems for computational processes to be helpful and work with the development of a fuzzy neuron based on the understanding that the structure of biological neurons obtained sociology begins and then learning machines are created that lead to these actions three-stage process in a fuzzy arithmetic - to be nervous is given below:

- Development of fuzzy neural models that are motivated by biological neurons

- Models of synaptic communication within the building that fuzzy neural networks combined

- develop learning algorithms (which at this stage the synaptic weights are adjusted) Using a combination of smart successful applications in areas including processes, engineering, business - financial, value added, medical diagnosis and cognitive simulation is growing rapidly.

\section{Routing with fuzzy method}

Fuzzy methods used in mobile robots

1 - Position control robot to reach target

2 - Strategies to avoid collision with obstacles

3 - To trace the route

4 - Navigation

5 - Route planning

6 - Fuzzy mobile robot model

7 - To follow the dynamic route

8 - Wall pursued the issue

9 - Control of mobile robot reference model variables

\section{Avoiding collision with obstacles}

For the problem to avoid dealing with the obstacles that arose the idea of fuzzy image method controls the behavior of reaction is used. Obstacle when the robot face left or right shift gives. For the robot could start to end point without the barrier to reach self, it is necessary at any time input data from the environment to receive the following? 
Distance to the target robot

Angle between the target robots

Distance to prevent the robot

Angle between the robots to stop

Regulation that is used for this purpose is as follows:

RuleR $^{i}:$ IF $\rho_{t} i s A_{1} A N D \theta_{t} i s B_{1} A N D \rho_{0} i s A_{2} A N D \theta_{0} i s B_{2}$ then $i s n^{i}$,

Goal is necessary to control a fuzzy controller to reach the barrier obtained with the inference engine control in method position be considered. The controller must produce practical that when faced with the obstacle away. These can create a negative angle hath required for robot guidance is the obstacle.

$$
\gamma_{0}(K)=F\left[\rho_{0}(K), \theta_{0}(K)\right]=-n_{0} .
$$

Diverging to prevent this situation from the point of aim, robot angle hath final combination of the two acts is as follows:

First fuzzy controller, the angle required for hath guided the robot to the target is calculated.

Then the fuzzy controller, a negative angle required for the guidance hath robot calculates the barrier.

$$
\begin{aligned}
& \gamma(k)=\gamma_{t}(k)+\gamma_{0}(k) \\
& \text { Or }
\end{aligned}
$$

$\gamma(k)=m_{t}-n_{0}$,

The Model to avoid dealing with the barrier becomes active when the following two conditions are satisfied:

$$
\begin{aligned}
& d \leq d_{s}, \\
& |\theta| \leq \theta_{s},
\end{aligned}
$$

The effect of distance and direction is the effect of area. Quantity of these two diagnostic areas that is cone shaped sets (according figure 7).
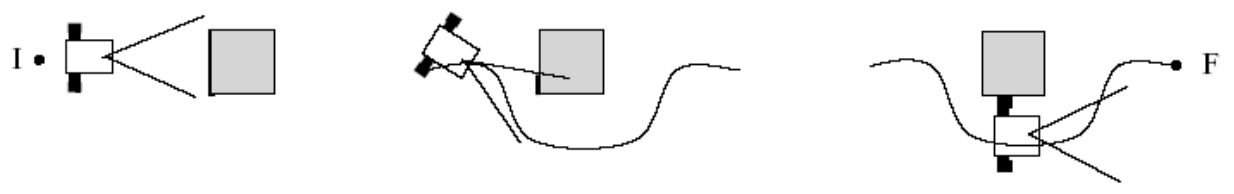

Figure 7. Effect of distance and direction area

Any object that was issued from the cone area, the robot can be located by sensors installed in front of the robot is detected. Fuzzy controller obstacle when the robot is active detection area is entered. When dealing with risk there is no obstacle, the controller has been disabled to prevent and control the robot only influenced target moves toward the goal. To obtain the proper angle without fuzz building height is used.

$$
\gamma(k)=C_{F}\left[\frac{\sum_{i=1}^{L} \mu_{i} m_{i}}{\sum_{i=1}^{L} \mu_{i}}-\frac{\sum_{j=1}^{M} \mu_{j} m_{j}}{\sum_{j=1}^{M} \mu_{j}}\right] .
$$

Advantages compared with other ways to pursue a dynamic way:

1 - Fuzzy controller, the robot moves along a path that can be broken by some high-level optimization algorithms to calculate, controls 
2 - Position of control over parts of the robot has been successfully until the desired position is that the logic path last point is achieved.

3 - Although fuzzy logic controller based on precise mathematical model has been done, this control is robust and flexible $[10,11]$.

\section{Conclusion}

Considering the importance of routing, algorithms mentioned above, all examples of data mining algorithms for this purpose are used. We provide complete solutions to support robot navigation and learning more efficient and they can make practical field research and subsequent activities.

\section{References}

[1] Jamal Shahrasbi doctor, secretary of Iran _ Conference on "Data Mining Engineer" Ali Zolghadr brave, MSc Industrial Engineering, Printing, Publishing Jihad University, 1388

[2] Engineer Mehdi Ali, "an introduction to genetic algorithms and its applications "Zanys Publications, Third Printing, 1387

[3] Lale zbakir, Adil Baykasoglu, Pınar Tapkan,"Bees algorithm for generalized assignment problem", Applied Mathematics and Computation 215 (2010) 3782-3795

[4] Mehdi Ghatee, Ali Mohades "Motion planning in order to optimize the length and clearance applying a Hopfield neural network", Expert Systems with Applications 36 (2009)

[5] Q. Zhang et al. "Gradient-based target localization in robotic sensor networks", Pervasive and Mobile Computing 5 (2009)

[6] Jeffery W. Seifert, "Analyst in information science and Technology Policy Data Mining: An Overview" ‘ December 2004

[7] Aghajani, M. "Application of Artificial Neural Network in distillation column Identification". Ms.Thesis of Shiraz University, Iran (2001)

[8] yahya mohamadi,Mohamad Hassan bahari" Routing with ant algorithm",(2000)

[9] M. B. Menhaj, "Fundamentals of Neural Networks". Amir Kabir University, Iran (1999)

[10] www.civilica.com.

[11] www.farsiyab.info. 